Canad. J. Math. Vol. 65 (6), 2013 pp. 1201-1216

http://dx.doi.org/10.4153/CJM-2012-031-3

(c) Canadian Mathematical Society 2012

\title{
Application of the Strong Artin Conjecture to the Class Number Problem
}

\author{
Peter J. Cho and Henry H. Kim
}

Abstract. We construct unconditionally several families of number fields with the largest possible class numbers. They are number fields of degree 4 and 5 whose Galois closures have the Galois group $A_{4}, S_{4}$, and $S_{5}$. We first construct families of number fields with smallest regulators, and by using the strong Artin conjecture and applying the zero density result of Kowalski-Michel, we choose subfamilies of $L$-functions that are zero-free close to 1 . For these subfamilies, the $L$-functions have the extremal value at $s=1$, and by the class number formula, we obtain the extreme class numbers.

\section{Introduction}

Let $\mathfrak{K}\left(n, G, r_{1}, r_{2}\right)$ be the set of number fields of degree $n$ with signature $\left(r_{1}, r_{2}\right)$ whose normal closures have $G$ as their Galois group. Then by the class number formula, the class number $h_{K}$ for $K \in \mathfrak{K}\left(n, G, r_{1}, r_{2}\right)$ is given by

$$
h_{K}=\frac{w_{K}\left|d_{K}\right|^{\frac{1}{2}}}{2^{r_{1}}(2 \pi)^{r_{2}} R_{K}} L(1, \rho),
$$

where $w_{K}$ is the number of roots of unity in $K, d_{K}$ is the discriminant of $K, R_{K}$ is its regulator, and $L(s, \rho)=\zeta_{K}(s) / \zeta(s)$ is the Artin L-function.

If $K$ has at least one real embedding, $w_{K}=2$. If $K$ has no real embedding, then $\phi\left(w_{K}\right) \leq n$. Since $\phi(m) \geq \sqrt{m}$, if $m \neq 2,6$ (cf. [20, p. 9]), $w_{K} \leq 4 n^{2}$.

Silverman [23] obtained a lower bound of the regulator $R_{K}$ of number fields $K$ :

$$
R_{K}>c_{n}\left(\log \gamma_{n}\left|d_{K}\right|\right)^{r-r_{0}}
$$

where $c_{n}, \gamma_{n}$ are positive constants depending on the degree $n$ of $K, r=r_{1}+r_{2}-1$, and $r_{0}$ is the maximum of unit ranks of subfields of $K$.

It is easy to prove that under the Artin conjecture and Generalized Riemann Hypothesis (GRH) for $L(s, \rho), L(1, \rho) \ll\left(\log \log \left|d_{K}\right|\right)^{n-1}$. (See the Remark 2.2.) Hence we obtain the conjectural upper bound for the class numbers

$$
h_{K} \ll\left|d_{K}\right|^{\frac{1}{2}} \frac{\left(\log \log \left|d_{K}\right|\right)^{n-1}}{\left(\log \left|d_{K}\right|\right)^{r-r_{0}}} .
$$

Received by the editors May 1, 2012.

Published electronically September 8, 2012.

H. Kim partially supported by an NSERC grant.

AMS subject classification: 11R29, 11M41.

Keywords: class number, strong Artin conjecture. 
Here the implied constants depend on $n$. Now the question is whether the upper bound is sharp. Namely, are there number fields with the largest possible class number of the size

$$
\left|d_{K}\right|^{\frac{1}{2}} \frac{\left(\log \log \left|d_{K}\right|\right)^{n-1}}{\left(\log \left|d_{K}\right|\right)^{r-r_{0}}} ?
$$

For real quadratic fields, this is a classical result of Montgomery and Weinberger [14]. Ankeny, Brauer, and Chowla [1] constructed unconditionally, for any $n, r_{1}, r_{2}$, number fields with arbitrarily large discriminants and $h_{K} \gg\left|d_{K}\right|^{1 / 2-\epsilon}$. Under the GRH and Artin conjecture for $L(s, \rho)$, Duke [6] constructed totally real fields of degree $n$ whose Galois closures have the Galois group $S_{n}$ with the largest possible class numbers. Daileda [7] showed Duke's result unconditionally when $n=3$, and Cho [2] showed it when $n=4$. He also proved that the Strong Artin Conjecture can replace the hypotheses of the Artin conjecture and the GRH in [6] when $n \geq 5$.

In this paper, we construct unconditionally a family of number fields with the largest possible class numbers. Namely,

(1) $n=5, G=S_{5},\left(r_{1}, r_{2}\right)=(1,2)$,

(2) $n=4, G=S_{4},\left(r_{1}, r_{2}\right)=(2,1)$,

(3) $n=4, G=S_{4},\left(r_{1}, r_{2}\right)=(0,2)$,

(4) $n=4, G=A_{4},\left(r_{1}, r_{2}\right)=(0,2)$.

Note that in all these cases, there are no nontrivial subfields. So $r_{0}=0$; it is clear when $n=5$. If $G=A_{4}$, it is clear, since $A_{4}$ does not have a subgroup of order 6. If $G=S_{4}$, the subgroup of order 12 is $A_{4}$, but it does not contain a subgroup isomorphic to $S_{3}$.

The family of number fields will be obtained as $K_{t}=\mathbb{Q}\left[\theta_{t}\right]$, where $\theta_{t}$ is a root of $f(x, t)=x^{n}+a_{1}(t) x^{n-1}+\cdots+a_{n}(t) \in \mathbb{Z}[t][x]$. As we see in the class number formula, we first need to construct a family of number fields with the smallest regulators, namely, $R_{K} \ll\left(\log \left|d_{K}\right|\right)^{r}$, and then find a subfamily for which $L(1, \rho) \gg$ $\left(\log \log \left|d_{K}\right|\right)^{n-1}$. Under the assumption of the GRH of $L(s, \rho)$, this can be done by carefully analyzing the set of primes that split completely. Here we need the concept of regular extensions over $\mathbb{Q}(t)$ and use the result of Serre [22] that says that given a regular extension, with a specialization $t \in \mathbb{Z}$, we can choose a subfamily in which almost all small primes split completely. We also need to study square-free and cubefree values of certain polynomials in arithmetic progressions. In particular, in the $A_{4}$ case, we need the fact that the number of $1 \leq t<X, t \equiv t_{M}(\bmod M)$ such that $1+27 t^{4}$ is cube-free, is $c \frac{X}{M}+O\left(X / M\left(\log \frac{X}{M}\right)^{3 / 5}\right)$ for some constant $c$. This follows easily from the result of Hooley [8, p. 69].

In the absence of the GRH, we have to use the zero density result of [13] to show that in a family of automorphic $L$-functions, every $L$-function outside a negligible set is zero-free in a desired region. For this, we need to show that $L(s, \rho)$ is an automorphic $L$-function of $G L_{n-1} / \mathbb{Q}$. This is called the Strong Artin Conjecture. For the case where $G=S_{5}$, Calegari [4] showed the modularity of $\rho$. In the case of $G=A_{4}$ (resp. $\left.S_{4}\right), \rho$ is equivalent to a twist of $\operatorname{Sym}^{2}(\sigma)$ by a character, where $\sigma$ is the 2-dimensional representation of $\widetilde{A}_{4} \simeq S L_{2}\left(\mathbb{F}_{3}\right)$, (resp. $\left.\widetilde{S}_{4} \simeq G L_{2}\left(\mathbb{F}_{3}\right)\right)$. So $\rho$ is modular. (See [2] for details.) 
Here we note that $L(1, \rho) \gg\left(\log \log \left|d_{K}\right|\right)^{n-1}$ does not hold when the number field does not come from regular extensions. For example, Daileda [7] showed that for a pure cubic extension given by $f(x, t)=x^{3}-t, L(1, \rho) \ll \log \log \left|d_{K}\right|$.

In a separate paper [3], we study dihedral and cyclic extensions with large class numbers. In this case, the representation $\rho$ is no longer attached to a cuspidal automorphic representation. We modify the result of [13] to apply in this case. Also, the existence of subfields makes it difficult to obtain number fields with the sharp bound (1.2).

We have not been able to find a family of quintic extensions in $\mathfrak{K}\left(5, A_{5}, 1,2\right)$ with the smallest possible regulators. (See Remark 8.6.) We also note that if the discriminant is positive, the number of complex roots of a polynomial in $\mathbb{R}[X]$ is a multiple of 4. Hence there are no number fields of type $\mathfrak{K}\left(5, A_{5}, 3,1\right)$ and $\mathfrak{K}\left(4, A_{4}, 2,1\right)$. It would be of interest to construct totally real number fields whose Galois closures have $A_{4}$ and $A_{5}$ as their Galois groups with the largest possible class numbers.

\section{Approximation of $L(1, \rho)$ and Zero density Result}

We use the following result of Daileda [7], giving the approximation of $\log L(1, \rho)$ as a sum over small primes. Let $\rho$ be an $l$-dimensional complex representation of a Galois group. We assume that $L(s, \rho)$ is an entire Artin L-function and let $N$ be its conductor. Also, $L(s, \rho)$ has a Dirichlet series

$$
L(s, \rho)=\sum_{n=1}^{\infty} \lambda(n) n^{-s}
$$

Proposition 2.1 ([7]) Let $L(s, \rho)$ and $N$ be as above. Let $6 / 7<\alpha<1$. Suppose that $L(s, \rho)$ is zero-free in the rectangle $[\alpha, 1] \times\left[-(\log N)^{2},(\log N)^{2}\right]$. If $N$ is sufficiently large, then for any $0<k<16 /(1-\alpha)$,

$$
\log L(1, \rho)=\sum_{p \leqslant(\log N)^{k}} \lambda(p) p^{-1}+O_{l, k, \alpha}(1)
$$

Remark 2.2 This implies immediately under GRH that $L(1, \rho) \ll(\log \log N)^{l}$, since $|\lambda(p)| \leq l$ and $\sum_{p \leq x} 1 / p=\log \log x+O(1)$.

Due to lack of the GRH, we cannot use the above result directly. We use the following zero density result of Kowalski-Michel to show that in a family of automorphic $L$-functions, every $L$-function outside a negligible set is zero-free in a desired region. Let $n \geqslant 1$ be a fixed integer. For all $q \geqslant 1$, let $S(q)$ be a finite set of cuspidal automorphic representations of $G L_{n} / \mathbb{Q}$ that satisfy the following conditions:

1. The forms $f \in S(q)$ satisfy the Ramanujan-Petersson conjecture at the finite places.

2. There exists $e>0$ such that for all $f \in S(q)$, the conductor Cond $(f)$ of $f$ satisfies

$$
\operatorname{Cond}(f) \leqslant q^{e}
$$


3. There exists $d>0$ such that $|S(q)| \ll q^{d}$ for all $q \geqslant 1$, the implied constant depending on the family.

4. All the $f \in S(q)$ have the same component at $\infty$, hence the same gamma factor in the functional equation.

For any cuspidal representation $f$ on $G L_{n} / \mathbb{Q}, \alpha \in \mathbb{R}$, and $T \geq 0$, we let

$$
N(f, \alpha, T)=|\{\rho|L(f, \rho)=0, \operatorname{Re}(\rho) \geq \alpha,| \operatorname{Im}(\rho) \mid \leq T\}|
$$

(zeros counted with multiplicity).

Proposition $2.3([13]) \quad$ Let $c_{0}$ be a constant with $c_{0}>5 n e / 2+d$. Let $\alpha \geqslant 3 / 4$ and $T \geqslant 2$. Then

$$
\sum_{f \in S(q)} N(f, \alpha, T) \ll T^{B} q^{c_{0} \frac{1-\alpha}{2 \alpha-1}}
$$

for all $q \geqslant 1$ and some $B \geqslant 0$ (depending on the family). The implied constant depends only on the family.

\section{Regular Extensions and their Galois Representations}

A finite Galois extension $E$ of the rational function field $\mathbb{Q}(t)$ is called regular if $\overline{\mathbb{Q}} \cap$ $E=\mathbb{Q}$. This is equivalent to the fact that $\operatorname{Gal}(E \overline{\mathbb{Q}} / \overline{\mathbb{Q}}(t)) \simeq \operatorname{Gal}(E / \mathbb{Q}(t))$. Suppose that

$$
f(x, t)=x^{n}+a_{1}(t) x^{n-1}+\cdots+a_{n}(t) \in \mathbb{Z}[t][x]
$$

is an irreducible polynomial of degree $n$ and gives rise to a regular Galois extension over $\mathbb{Q}(t)$ with the Galois group $G$. Let $K_{t}$ be a field obtained by adjoining to $\mathbb{Q}$ a root of $f(x, t)$ with a specialization $t \in \mathbb{Z}$ and let $\widehat{K}_{t}$ be the Galois closure of $K_{t}$. Let $C$ be any conjugacy class of $G$. Serre observed the following important fact, regarding distribution of Frobenius elements in a regular Galois extension [22, p. 45].

Theorem 3.1 There is a constant $c_{f}>0$ depending on $f$ such that for any prime $p \geq c_{f}$, there is $t_{C} \in \mathbb{Z}$ so that for any $t \equiv t_{C}(\bmod p)$, $p$ is unramified in $\widehat{K}_{t} / \mathbb{Q}$, and $\operatorname{Frob}_{p} \in C$.

We write

$$
L(s, \rho, t)=\frac{\zeta_{K_{t}}(s)}{\zeta(s)},
$$

where $\rho: G \rightarrow G L_{n-1}(\mathbb{C}), H=\operatorname{Gal}\left(\widehat{K}_{t} / K_{t}\right)$, and $\operatorname{Ind}_{H}^{G} 1_{H}=1_{G} \oplus \rho$.

Conjecture 3.2 (Strong Artin Conjecture) Let $\rho$ be as above. Then $\rho$ is modular, namely, $L(s, \rho, t)$ is an automorphic $L$-function of $G L_{n-1} / \mathbb{Q}$.

\section{Extreme Class Numbers}

In this section, we explain how to obtain the extreme class numbers in a general setting. Let $G$ be a finite group and let $f(x, t) \in \mathbb{Z}[t][x]$ be an irreducible polynomial 
of degree $n$ whose splitting field over $\mathbb{Q}(t)$ is a regular extension with Galois group $G$. Let $K_{t}, \widehat{K}_{t}$, and $L(s, \rho, t)$ be as in Section 3. The conductor of $L(s, \rho, t)$ is $\left|d_{K_{t}}\right|$. Let

$$
L(s, \rho, t)=\sum_{n=1}^{\infty} \lambda_{t}(n) n^{-s}
$$

where $\lambda_{t}(p)=N_{t}(p)-1$ and $N_{t}(p)$ is the number of solutions of $f(x, t) \equiv 0$ $(\bmod p)$. Hence, $-1 \leq \lambda_{t}(p) \leq n-1$.

Here we restrict $\rho$ to be irreducible in order to apply Proposition 2.3. If $G$ is dihedral or cyclic, $\rho$ is no longer irreducible. In this case, we need to modify Proposition 2.3. We treat this case in a forthcoming paper [3].

We assume Conjecture 3.2 (the Strong Artin Conjecture) for $L(s, \rho, t)$. We expect that the regular Galois extension property implies that the absolute value of a field discriminant will increase with respect to $t$ with a specialization $t \in \mathbb{Z}$.

Assumption 4.1 If $|t|$ is sufficiently large, $\log \left|d_{K_{t}}\right| \gg_{f} \log |t|$.

By the class number formula (1.1) and regulator bound (1.2), we first need to construct a family of number fields with the smallest regulators $R_{K_{t}} \ll\left(\log \left|d_{K_{t}}\right|\right)^{r-r_{0}}$. Next, we need to make $L(1, \rho, t)$ largest possible. In light of Proposition 2.1, we need to choose $t$ such that $\lambda_{t}(p)=n-1$ for almost all $p \leqslant\left(\log \left|d_{K_{t}}\right|\right)^{k}$ for some $k$. Namely, we need to choose $t$ such that almost all $p \leqslant\left(\log \left|d_{K_{t}}\right|\right)^{k}$ split completely in $K_{t}$.

Since $f(x, t)$ gives rise to a regular extension over $\mathbb{Q}(t)$, by Theorem 3.1 , there is an integer $c_{f}$ such that for all prime numbers $q \geq c_{f}$, there is an integer $t_{q}$ so that for any $t \equiv t_{q}(\bmod q), q$ splits completely in $\widehat{K}_{t}$. Now, for given $X \gg 0$, define

$$
y=\frac{\log X}{\log \log X} \quad \text { and } \quad M=\prod_{c_{f} \leq q \leq y} q .
$$

Let $t_{M}$ be an integer such that $t_{M} \equiv t_{q}(\bmod q)$ for all $c_{f} \leq q \leq y$. Here $\log M \sim y$, and hence $M \ll X^{\epsilon}$ for any $\epsilon>0$.

Assume that the discriminant of $f(x, t)$ is a polynomial in $t$ of degree $D$, and $\left|d_{K_{t}}\right| \leq C t^{D}$ for some constant $C$. We define a set $L(X)$ of positive numbers given by

$$
L(X)=\left\{\frac{X}{2}<t<X \mid t \equiv t_{M}(\bmod M), \operatorname{Gal}\left(\widehat{K}_{t} / \mathbb{Q}\right) \simeq G\right\} .
$$

Under the Strong Artin Conjecture, every $t$ in $L(A)$ gives rise to an automorphic L-function of $G L_{n-1}$ over $\mathbb{Q}$. However, it is possible that different $t$ in $L(A)$ may give rise to the same $L$-function, namely, $\zeta_{K_{t_{1}}}(s)=\zeta_{K_{t_{2}}}(s)$. In that case, we say that $K_{t_{1}}$ and $K_{t_{2}}$ are arithmetically equivalent. We make the following assumption.

Assumption 4.2 There exists an integer $m>0$, depending only on $f$, such that there are at most $m$ values of $t$ giving rise to the same L-function.

In order to verify the assumption, we use the following theorem. 
Theorem 4.3 (Klingen [12]) Let $K / \mathbb{Q}$ be a number field of degree $n \leq 11$. Let $\widehat{K}$ be the Galois closure and assume that there exists a non-conjugate field $K^{\prime}$ which is arithmetically equivalent to $K$. Then up to conjugacy, only the following 4 cases are possible for $G=\operatorname{Gal}(\widehat{K} / \mathbb{Q})$ :

(i) $n=7, G=G L_{3}(2)$;

(ii) $n=8, G=\mathbb{Z} / 8 \mathbb{Z} \rtimes(\mathbb{Z} / 8 \mathbb{Z})^{\times}$;

(iii) $n=8, G=G L_{2}(3)$;

(iv) $n=11, G=P S L_{2}(11)$.

We will prove that there exists $m>0$ such that there are at most $m$ isomorphic $K_{t}$ 's for $t \in L(X)$. Hence by the above theorem, they are not arithmetically equivalent and Assumption 4.2 is verified.

Let $\widetilde{L}(X)$ be the set of automorphic L-functions coming from $L(X)$ after removing the possible repetition of the same $L$-functions among them. In Sections 5 through 8 , we consider explicit examples of families of number fields. In those cases, we may have to put more conditions in $L(X)$ in order to satisfy Assumption 4.2, or replace it by some other set. In any case, we show that $X^{1-\epsilon} \ll|\widetilde{L}(X)| \ll X$ for any fixed $\epsilon>0$.

Let $c_{0}=5(n-1) D / 2+1$. Choose $\alpha$ with $c_{0}(1-\alpha) /(2 \alpha-1)<98 / 100$. By applying Proposition 2.3 to $\widetilde{L}(X)$ with $e=D, d=1$, and $T=\left(\log C X^{D}\right)^{2}$, every automorphic $L$-function excluding exceptional $O\left(X^{98 / 100}\right) L$-functions has a zerofree region $\left.[\alpha, 1] \times\left[-\left(\log \left|d_{K_{t}}\right|\right)^{2}, \log \left|d_{K_{t}}\right|\right)^{2}\right]$. Let us denote by $\widehat{L}(X)$ the set of the automorphic $L$-functions with the zero-free region.

Applying Proposition 2.1 to $L$-functions in $\widehat{L}(X)$, we have

$$
\begin{aligned}
\log L(1, \rho, t) & =\sum_{q \leqslant\left(\log \left|d_{K_{t}}\right|\right)^{1 / 2}} \lambda_{t}(q) q^{-1}+O_{n, \alpha}(1) \\
& =(n-1) \sum_{c_{f} \leqslant q \leqslant\left(\log \left|d_{K_{t}}\right|\right)^{1 / 2}} q^{-1}+O_{n, \alpha}(1) \\
& =(n-1) \log \log \log \left|d_{K_{t}}\right|+O_{n, \alpha}(1)
\end{aligned}
$$

where we use the fact that $\left(\log \left|d_{K_{t}}\right|\right)^{1 / 2} \leqslant y=\log X / \log \log X$ for large $X$. So we have $L(1, \rho, t) \gg\left(\log \log \left|d_{K_{t}}\right|\right)^{n-1}$. Hence we have the required result

$$
h_{K_{t}} \gg\left|d_{K_{t}}\right|^{\frac{1}{2}} \frac{\left(\log \log \left|d_{K_{t}}\right|\right)^{n-1}}{\left(\log \left|d_{K_{t}}\right|\right)^{r-r_{0}}} .
$$

\section{$5 S_{5}$ Extensions with Signature $(\mathbf{1}, \mathbf{2})$}

Let

$$
f(x, t)=(x+t)\left(x^{2}+5 t\right)\left(x^{2}+10 t\right)+t
$$

with the discriminant

$$
\begin{aligned}
& \operatorname{Disc}(f(x, t))=t^{4}\left(500000 t^{10}+15000000 t^{9}+162350000 t^{8}+746700000 t^{7}\right. \\
& \left.\quad+1234759600 t^{6}+7714500 t^{5}-394744 t^{4}+5143500 t^{3}+162500 t^{2}+3125\right) .
\end{aligned}
$$


For a non-zero integer $t, f(x, t)$ has one real root and four complex roots.

We claim that the Galois groups of $f(x, t)$ over $\mathbb{Q}(t)$ and $\overline{\mathbb{Q}}(t)$ are both $S_{5}$. Since $f(x, t)$ is an Eisenstein polynomial for an irreducible element $t$, it is irreducible over $\mathbb{Q}(t)$ and $\overline{\mathbb{Q}}(t)$ and it is clear that $\operatorname{Disc}(f(x, t))$ is not an square in $\mathbb{Q}(t)$ and $\overline{\mathbb{Q}}(t)$. If the sextic resolvent has no root in $\mathbb{Q}(t)$ and $\overline{\mathbb{Q}}(t)$, then the Galois group is $S_{5}$ over both fields. The sextic resolvent of $f(x, t)$ is given by

$$
\theta_{t}(y)=\left(y^{3}+b_{2} y^{2}+b_{4} y+b_{6}\right)^{2}-2^{10} \operatorname{Disc}(f(x, t)) y
$$

where

$$
\begin{gathered}
b_{2}=5 t^{2}(24 t-335), \quad b_{4}=t^{3}\left(400 t^{3}-192000 t^{2}+661811 t-2400\right), \\
b_{6}=5^{2} t^{3}\left(12400 t^{5}+3069000 t^{4}+17775 t^{3}+168480 t^{2}-64 t+2400\right) .
\end{gathered}
$$

If $\alpha$, a divisor of $b_{6}^{2}$, is a root of $\theta_{t}(y)$, then

$$
\left(\alpha^{3}+b_{2} \alpha^{2}+b_{4} \alpha+b_{6}\right)^{2}=2^{10} \operatorname{Disc}(f(x, t)) \alpha .
$$

Since the RHS of (5.1) cannot be a square, it is a contradiction. Hence $f(x, t)$ gives rise to an $S_{5}$ regular extension over $\mathbb{Q}(t)$.

Recently, Calegari obtained the modularity of $S_{5}$ Galois representations for a special case.

Theorem 5.1 (Calegari $[4]) \quad$ Let $K / \mathbb{Q}$ be a quintic extension with $\operatorname{Gal}(\widehat{K} / \mathbb{Q})=S_{5}$. Furthermore, we assume that

(i) the complex conjugation in $\mathrm{Gal}(\widehat{K} / \mathbb{Q})=S_{5}$ has the conjugacy class $(12)(34)$;

(ii) the extension $\widehat{K} / \mathbb{Q}$ is unramified at 5 and the Frobenius element Frob ${ }_{5}$ has the conjugacy class $(12)(34)$.

If $\rho: \operatorname{Gal}(\widehat{K} / \mathbb{Q}) \rightarrow G L_{4}(\mathbb{C})$ is an irreducible representation of dimension 4 , then $\rho$ is modular.

Remark 5.2 Calegari observed that the 4-dimensional representation $\rho$ is equivalent to a twist of $A s(\sigma)$ by a character, where $\sigma$ is the 2-dimensional icosahedral representation of $\widetilde{A}_{5}$ over the quadratic subextension $F$ and $A s$ is the Asai lift. He then used the modularity of $\sigma$ proved by Sasaki [19]. In his thesis [24], Y. Zhang also observed the fact that $\rho$ is twist equivalent to $A s(\sigma)$.

Let $K_{t}=\mathbb{Q}\left(\theta_{1}\right)$ be a quintic field obtained by adjoining the real root $\theta_{1}$ of $f(x, t)$ to the rational number field $\mathbb{Q}$. We assume that $t \equiv 1(\bmod 5)$. Then

$$
f(x, t) \equiv x^{4}(x+1)+1 \equiv(x+2)\left(x^{2}+x+1\right)\left(x^{2}+3 x+3\right)(\bmod 5)
$$

and the signature of $K_{t}$ is $(1,2)$. Hence the Galois extensions $\widehat{K}_{t} / \mathbb{Q}$ satisfy the hypotheses of Theorem 5.1, and Artin L-functions $L(s, \rho, t)=\zeta_{K_{t}}(s) / \zeta(s)$ are cuspidal automorphic L-functions of $G L_{4} / \mathbb{Q}$. 
We claim that $\left(\theta_{1}+t\right)^{5} / t$ and $\left(\theta_{1}^{2}+5 t\right)^{5} / t^{2}$ are two independent units in $K_{t}$. Since $f(x, t)=x^{5}+t x^{4}+15 t x^{3}+15 t^{2} x^{2}+50 t^{2} x+50 t^{3}+t$

$$
\frac{\theta_{1}^{5}}{t}=-\left(\theta_{1}^{4}+15 \theta_{1}^{3}+15 t \theta_{1}^{2}+50 t \theta_{1}+50 t^{2}+1\right)
$$

Hence $\theta_{1}^{5} / t$ is an algebraic integer. From this, it is easy to show that $\left(\theta_{1}+t\right)^{5} / t$ and $\left(\theta_{1}^{2}+5 t\right)^{5} / t^{2}$ are algebraic integers. Now we have

$$
\frac{\left(\theta_{1}+t\right)^{5}}{t} \cdot \frac{\left(\theta_{1}^{2}+5 t\right)^{5}}{t^{2}} \cdot \frac{\left(\theta_{1}^{2}+10 t\right)^{5}}{t^{2}}=-1 .
$$

Hence $\left(\theta_{1}+t\right)^{5} / t$ and $\left(\theta_{1}^{2}+5 t\right)^{5} / t^{2}$ are units. To show that they are independent, we need to know the locations of 5 roots of $f(x, t)$. For the case of the real root $\theta_{1}$, we have $-t-1 / t<\theta_{1}<-t$. For complex roots, we use the following lemma from $[17$, p. 9]. See also [21].

Lemma 5.3 Let $f$ be a polynomial of degree $m$ and $f(\alpha) \neq 0, f^{\prime}(\alpha) \neq 0$. Then for every circle $C$ passing through $\alpha, \alpha-m f(\alpha) / f^{\prime}(\alpha)$, at least one root of $f$ is inside $C$, and one root outside of $C$.

We apply Lemma 5.3 to $f(x, t)$ with $\alpha=i \sqrt{5 t}$, then we can see that another root $\theta_{2}$ of $f(x, t)$ converges to $i \sqrt{5 t}$ as $t$ increases. More precisely,

$$
\left|\theta_{2}-i \sqrt{5 t}\right|=O\left(\frac{1}{t^{1.5}}\right)
$$

Put $\theta_{3}=\overline{\theta_{2}}$. Apply Lemma 5.3 again to $f(x, t)$ with $\alpha=i \sqrt{10 t}$. Then we can find the fourth $\operatorname{root} \theta_{4}$ with

$$
\left|\theta_{4}-i \sqrt{10 t}\right|=O\left(\frac{1}{t^{1.5}}\right)
$$

and put $\theta_{5}=\overline{\theta_{4}}$.

Assume that $\left(\theta_{1}+t\right)^{5} / t$ and $\left(\theta_{1}^{2}+5 t\right)^{5} / t^{2}$ are dependent. Then $\left(\left(\theta_{1}+t\right)^{5} / t\right)^{k}=$ $\left(\left(\theta_{1}^{2}+5 t\right)^{5} / t^{2}\right)^{l}$ for some integers $k, l$. Then it holds when we replace $\theta_{1}$ by $\theta_{4}$, and when we consider the size of $\theta_{1}, \theta_{4}$, we obtain a contradiction. By definition,

$$
R_{K_{t}} \ll\left|\operatorname{det}\left(\begin{array}{ll}
\log \left|\frac{\left(\theta_{1}+t\right)^{5}}{t}\right| & \log \left|\frac{\left(\theta_{4}+t\right)^{5}}{t}\right| \\
\log \left|\frac{\left(\theta_{1}^{2}+5 t\right)^{5}}{t^{2}}\right| & \log \left|\frac{\left(\theta_{4}^{2}+5 t\right)^{5}}{t^{2}}\right|
\end{array}\right)\right| .
$$

By the above estimates on $\theta_{1}, \theta_{4}$, it is clear that $R_{K_{t}} \ll(\log t)^{2}$. Since $f(x, t)$ is an Eisenstein polynomial, $d_{K_{t}}$ is divisible by $t^{4}$ if $t$ is square-free. (See [16, p. 60].) Hence $\log d_{K_{t}} \gg \log t$, and Assumption 4.1 is verified. We have proved the following lemma.

Lemma 5.4 For a square-free positive integer $t, R_{K_{t}} \ll\left(\log d_{K_{t}}\right)^{2}$. 
As described in Section 4, we define a set $L(X)$ of square-free integers:

$$
L(X)=\left\{\frac{X}{2}<t<X \mid t \equiv t_{M}(\bmod M) \text { and } t \text { square-free }\right\} .
$$

Each $t$ in $L(X)$ gives rise to an automorphic L-function $L(s, \rho, t)=\zeta_{K_{t}}(s) / \zeta(s)$ of $G L_{4} / \mathbb{Q}$. We claim that for a square-free integer $t$,

$$
p \text { is totally ramified in } K_{t} \Longleftrightarrow p \text { divides } t \text {. }
$$

It is known that those primes dividing $t$ totally ramify in $K_{t}$. (See [5, Corollary 6.2.4].) Assume that $p$ totally ramifies but does not divide $t$. This means that $f(x, t) \equiv x^{5}$ or $(x+a)^{5}(\bmod p)$ for $p \nmid t$. We can induce a contradiction by comparing coefficients modulo $p$. Hence Assumption 4.2 is also verified.

On the other hand, by [7, p. 248], $|L(X)|=c \frac{X}{2 M}+O\left(X^{1 / 2}\right)$ where

$$
c=\frac{6}{\pi^{2}} \prod_{p \mid M}\left(1-p^{-2}\right)^{-1} \quad \text { and } \quad X^{1-\epsilon} \ll|L(X)| \ll X .
$$
lows:

Hence by the argument in Section 4, we have the result. We summarize it as fol-

Theorem 5.5 There is a constant $c>0$ such that there exist $K \in \mathfrak{K}\left(5, S_{5}, 1,2\right)$ with arbitrarily large discriminant $d_{K}$ for which

$$
h_{K}>c d_{K}^{\frac{1}{2}} \frac{\left(\log \log d_{K}\right)^{4}}{\left(\log d_{K}\right)^{2}} .
$$

\section{$6 \quad S_{4}$ Extensions with Signature $(2,1)$}

Let $t>1$ be a positive square-free integer and $f(x, t)=x^{2}(x-10 t)(x-18 t)+t$. Then the discriminant of $f(x, t)$ is

$$
\operatorname{Disc}(f(x, t))=-256 t^{3}(12 t+1)(15 t-1)\left(144 t^{2}-12 t+1\right)\left(225 t^{2}+15 t+1\right)<0 .
$$

Since $f(x, t)$ is an Eisenstein polynomial for each prime divisor of $t, d_{K_{t}}$ is divisible by $t^{3}$, and assumption 4.1 is verified. The cubic resolvent $y^{3}-180 t^{2} y^{2}-4 t y-64 t^{3}$ of $f(x, t)$ has the only real root between $180 t^{2}$ and $180 t^{2}+1$. Hence $f(x, t)$ gives rise to an $S_{4}$ Galois extension for each positive square-free integer $t$.

Consider $f(x, t)=x^{2}(x-10 t)(x-18 t)+t$ over $\overline{\mathbb{Q}}(t)$. It is easy to see that the cubic resolvent $y^{3}-180 t^{2} y^{2}-4 t y-64 t^{3}$, is irreducible over $\overline{\mathbb{Q}}[t]$. By Gauss' Lemma, it is irreducible over $\overline{\mathbb{Q}}(t)$. Hence the Galois group of $f(x, t)$ over $\overline{\mathbb{Q}}(t)$ is $S_{4}$. Therefore, $f(x, t)$ gives rise to a regular Galois extension over $\mathbb{Q}(t)$.

Note that $f^{\prime}(x, t)=4 x(x-6 t)(x-15 t)$, and we can see easily that $f(x, t)$ has two real roots $\theta_{1}, \theta_{2}$ and two complex roots $\theta_{3}, \theta_{4}=\overline{\theta_{3}}$. For sufficiently large $t$, we can see that $10 t+1 / t^{3}<\theta_{1}<10 t+1 / t^{2}, 18 t-1 / t^{2}<\theta_{2}<18 t-1 / t^{3}$. Also by taking $\alpha=1 / t$ and applying Lemma 5.3, we can see that $\theta_{3}$ and its conjugate $\theta_{4}$ are inside the circle of radius $1 / 9$ centered at the origin.

Let $K_{t}=\mathbb{Q}\left[\theta_{1}\right]$. Then we prove the following lemma. 
Lemma 6.1 $\theta_{1}^{4} / t$ and $\left(\theta_{1}-10 t\right)^{4} / t$ are independent units in $\mathbb{Z}_{K_{t}}$.

Proof Since $\theta_{1}^{4}-28 t \theta_{1}^{3}+180 t^{2} \theta_{1}^{3}+t=0, \theta_{1}^{4} / t=28 \theta_{1}^{3}-180 t \theta_{1}^{2}-1$. Hence $\theta_{1}^{4} / t$ is an algebraic integer. Also

$$
\begin{aligned}
\frac{\left(\theta_{1}-10 t\right)^{2}\left(\theta_{1}-18 t\right)^{2}}{t} & =\frac{\left(\theta_{1}^{2}-t\left(28 \theta_{1}-180 t\right)\right)^{2}}{t} \\
& =\frac{\theta_{1}^{4}}{t}-2 \theta_{1}^{2}\left(28 \theta_{1}-180 t\right)^{2}+t\left(28 \theta_{1}-180 t\right)^{2} .
\end{aligned}
$$

So $\left(\left(\theta_{1}-10 t\right)^{2}\left(\theta_{1}-18 t\right)^{2}\right) / t$ is an algebraic integer. Now we have

$$
\frac{\theta_{1}^{4}}{t} \cdot \frac{\left(\theta_{1}-10 t\right)^{2}\left(\theta_{1}-18 t\right)^{2}}{t}=1 .
$$

Hence $\theta_{1}^{4} / t$ is a unit. By considering $y=x-10 t$ or $y=x-18 t$, we can see that $\left(\theta_{1}-10 t\right)^{4} / t$ and $\left(\theta_{1}-18 t\right)^{4} / t$ are algebraic integers. We have

$$
\frac{\theta_{1}^{8}}{t^{2}} \cdot \frac{\left(\theta_{1}-10 t\right)^{4}}{t} \cdot \frac{\left(\theta_{1}-18 t\right)^{4}}{t}=1 .
$$

Hence $\left(\theta_{1}-10 t\right)^{4} / t$ is a unit.

Assume that $\theta_{1}^{4} / t$ and $\left(\theta_{1}-10 t\right)^{4} / t$ are dependent. Then

$$
\left(\frac{\theta_{1}^{4}}{t}\right)^{k}=\left(\frac{\left(\theta_{1}-10 t\right)^{4}}{t}\right)^{m}
$$

for some integers $k$ and $m$. Without loss of generality, we can assume that $k$ is positive. When we consider the size of $\theta_{1}, m$ should be a negative integer. But when we replace $\theta_{1}$ by $\theta_{2}, m$ should be a positive integer, which induces a contradiction.

Lemma 6.2 For positive square-free $t, R_{K_{t}} \ll\left(\log \left|d_{K_{t}}\right|\right)^{2}$.

Proof By definition,

$$
R_{K_{t}} \leq\left|\operatorname{det}\left(\begin{array}{cc}
\log \left|\frac{\theta_{1}^{4}}{t}\right| & \log \left|\frac{\theta_{2}^{4}}{t}\right| \\
\log \left|\frac{\left(\theta_{1}-10 t\right)^{4}}{t}\right| & \log \left|\frac{\left(\theta_{2}-10 t\right)^{4}}{t}\right|
\end{array}\right)\right| .
$$

By the above estimates on $\theta_{1}, \theta_{2}$, it is clear that $R_{K_{t}} \ll(\log t)^{2}$. Since $t^{3} \mid d_{K_{t}}$, we have proved the claim.

As described in Section 4, we construct a set $L(X)$ of square-free integers

$$
L(X)=\left\{\frac{X}{2}<t<X \mid t \text { square-free and odd, } t \equiv t_{M}(\bmod M)\right\} .
$$

As in the Section 5, we can see that $X^{1-\epsilon} \ll|L(X)| \ll X$.

Let $L(s, \rho, t)=\zeta_{K_{t}}(s) / \zeta(s)$. Then $\rho$ is equivalent to a twist of $\operatorname{Sym}^{2}(\sigma)$ by a character, where $\sigma$ is the 2-dimensional representation of $\widetilde{S}_{4} \simeq G L_{2}\left(\mathbb{F}_{3}\right)$. Hence $\rho$ is modular and $L(s, \rho, t)$ is a cuspidal automorphic $L$-function of $G L_{3} / \mathbb{Q}$. Hence Conjecture 3.2 is true for this case. See [2] for details.

Now we claim that Assumption 4.2 holds, as a consequence of the following lemma, which implies that $K_{t_{1}}$ and $K_{t_{2}}$ are not isomorphic if $t_{1} \neq t_{2}$. 
Lemma 6.3 For a square-free odd integer $t, p \neq 2$ is totally ramified in $K_{t}$ if and only if $p$ dividest.

Proof Since $f(x, t)$ is an Eisenstein polynomial, if $p \mid t, p$ is totally ramified. See [5, Corollary 6.2.4]. Conversely, suppose that $p$ is totally ramified and does not divide $t$. Then $f(x, t) \equiv(x+a)^{4}(\bmod p)$. By comparing the coefficients of $f(x, t)$ and $(x+a)^{4}(\bmod p)$, we obtain a contradiction.

We have shown that Assumptions 4.1, 4.2, and Conjecture 3.2 hold. Hence we can summarize our result as follows.

Theorem 6.4 There is a constant $c>0$ such that there exist $K \in \mathfrak{K}\left(4, S_{4}, 2,1\right)$ with arbitrarily large discriminant $d_{K}$ for which

$$
h_{K}>c\left|d_{K}\right|^{\frac{1}{2}} \frac{\left(\log \log \left|d_{K}\right|\right)^{3}}{\left(\log \left|d_{K}\right|\right)^{2}} .
$$

\section{$7 \quad S_{4}$ Extensions with Signature $(0,2)$}

Let $t>1$ be a positive square-free integer and $f(x, t)=x^{4}+t x^{2}+t x+t$. Then the discriminant $\operatorname{Disc}(f(x, t))$ of $f(x, t)$ is $t^{3}\left(12 t^{2}-11 t+256\right)$. Since $f(x, t)$ is an Eisenstein polynomial for each prime divisor of $t, d_{K_{t}}$ is divisible by $t^{3}$, hence Assumption 4.1 is verified.

The cubic resolvent $y^{3}-t y^{2}-4 t y+3 t^{2}$ of $f(x, t)$ has three real roots. One of them is located between $t+1$ and $t+2$, hence it is not an integer. So if the cubic resolvent has an integer root, we can show that the integer root should be divisible by $t$. Since $\pm t, \pm 3 t, \pm t^{2}$, and $\pm 3 t^{2}$ are not a root of the cubic resolvent, the cubic resolvent is irreducible. Hence $f(x, t)$ gives rise to an $S_{4}$ Galois extension for each positive square-free integer $t$.

Consider $f(x, t)=x^{4}+t x^{2}+t x+t$ over $\overline{\mathbb{Q}}(t)$. It is easy to see that the cubic resolvent $y^{3}-t y^{2}-4 t y+3 t^{2}$ is irreducible over $\overline{\mathbb{Q}}[t]$. By Gauss' Lemma, it is irreducible over $\overline{\mathbb{Q}}(t)$. Hence the Galois group of $f(x, t)$ over $\overline{\mathbb{Q}}(t)$ is $S_{4}$. Therefore, $f(x, t)$ gives rise to a regular Galois extension over $\mathbb{Q}(t)$.

Note that $f^{\prime}(x, t)=4 x^{3}+2 t x+t$ has only one real root $x_{0}$, and we can easily check that $f\left(x_{0}\right)>0$. Hence $f(x, t)$ has four complex roots $\theta_{1}, \theta_{2}=\overline{\theta_{1}}, \theta_{3}$, and $\theta_{4}=\overline{\theta_{3}}$. For sufficiently large $t$, when we apply Lemma 5.3 with $\alpha=i \sqrt{t}$, we can see that one root lies inside the circle of radius 1 centered at $1+i \sqrt{t}$. Let $\theta$ be the root.

Let $K_{t}=\mathbb{Q}[\theta]$. Then since $\theta^{4} / t=-\left(\theta^{2}+\theta+1\right), \theta^{4} / t$ is an algebraic integer. Here $N_{K_{t} / \mathbb{Q}}(\theta)=t$. Hence $\theta^{4} / t$ has norm 1 , and it is a unit in $\mathbb{Z}_{K_{t}}$.

Since $|\theta| \ll \sqrt{t}, \log \left|\theta^{4} / t\right| \ll \log t$. Since $t^{3} \mid d_{K_{t}}$, we have the following lemma.

Lemma 7.1 For positive square-free $t, R_{K_{t}} \ll \log d_{K_{t}}$.

As described in Section 4, we construct a set $L(X)$ of square-free integers,

$$
L(X)=\left\{\frac{X}{2}<t<X \mid t \text { square-free and } t \equiv t_{M}(\bmod M)\right\},
$$

and we can see that $X^{1-\epsilon} \ll|L(X)| \ll X$. 
Also, as in the previous sections, we can show that $p$ is totally ramified in $K_{t}$ if and only if $p \mid t$. Hence $K_{t_{1}}$ and $K_{t_{2}}$ are not isomorphic if $t_{1} \neq t_{2}$, and $L(s, \rho, t)$ 's are distinct. Therefore, Assumption 4.2 is verified, and we obtain the following theorem.

Theorem 7.2 There is a constant $c>0$ such that there exist $K \in \mathfrak{K}\left(4, S_{4}, 0,2\right)$ with arbitrarily large discriminant $d_{K}$ for which

$$
h_{K}>c d_{K}^{\frac{1}{2}} \frac{\left(\log \log d_{K}\right)^{3}}{\log d_{K}} .
$$

\section{$8 A_{4}$ Extensions with Signature (0,2)}

Consider $f(x, t)=x^{4}-8 t x^{3}+18 t^{2} x^{2}+1$, which is considered in [22, p. 44]. Note that $f^{\prime}(x, t)=4 x(x-3 t)^{2}$. Then $\operatorname{Disc}(f(x, t))=16^{2}\left(27 t^{4}+1\right)^{2}$. We claim that the splitting field of $f(x, t)$ over $\mathbb{Q}(t)$ is a regular extension with Galois group $A_{4}$. It is enough to show that the Galois group of $f(x, t)$ over $\overline{\mathbb{Q}}(t)$ is $A_{4}$. First, $f(x, t)$ is irreducible over $\overline{\mathbb{Q}}(t)$. By Gauss' Lemma, it is enough to check it over $\overline{\mathbb{Q}}[t]$. It is easy to check that $f(x, t)$ has no root in $\overline{\mathbb{Q}}[t]$. If $f(x, t)$ is a product of two quadratic polynomials, then

$$
x^{4}-8 t x^{3}+18 t^{2} x^{2}+1=\left(x^{2}+b x+c\right)\left(x^{2}+d x+\frac{1}{c}\right)
$$

for some $b, d \in \overline{\mathbb{Q}}[t]$ and $c \in \overline{\mathbb{Q}}$. We can induce a contradiction easily.

Its Ferrari resolvent $\theta(y)$ is $y^{3}-18 t^{2} y^{2}-4 y+8 t^{2}$, and it is irreducible over $\overline{\mathbb{Q}}[t]$. Since the discriminant of $f(x, t)$ is a square in $\overline{\mathbb{Q}}(t)$, the Galois group over $\overline{\mathbb{Q}}(t)$ is $A_{4}$.

We can see easily that $f(x, t)$ has 4 complex roots. If $\theta_{t}$ is a root, it is a unit. Let $K_{t}=\mathbb{Q}\left[\theta_{t}\right]$.

Proposition 8.1 The regulator $R_{K_{t}}$ satisfies $R_{K_{t}} \ll \log$.

Proof By considering $\alpha=6 t$ in Lemma 5.3, we can see that

$$
2 t-\frac{1}{54 t^{3}}<\left|\theta_{t}\right|<6 t
$$

Therefore, $R_{K_{t}} \leq 2 \log \left|\theta_{t}\right| \ll \log t$.

Note that $f(x, t)=(x+t)(x-3 t)^{3}+27 t^{4}+1$. Take $t$ such that $27 t^{4}+1$ is cubefree. Let $p \mid\left(27 t^{4}+1\right), p>3$. Then $p \nmid t$ and $f(x, t) \equiv(x+t)(x-3 t)^{3} \bmod p$. The vertices of the Newton polygon with respect to $x-3 t$ are $(0,0),(1,0),(4, i)$ with $i=1,2$. By Cohen $\left[5\right.$, p. 315], $p \mathbb{Z}_{K_{t}}=\mathfrak{p}_{1} \mathfrak{p}_{2}^{3}$, with prime ideals $\mathfrak{p}_{1}, \mathfrak{p}_{2}$. Hence $p \mid d_{K_{t}}$. Therefore, $d_{K_{t}} \geq \prod_{p \mid\left(27 t^{4}+1\right)} p$.

But $27 t^{4}+1 \leq\left(\prod_{p \mid\left(27 t^{4}+1\right)} p\right)^{2}$. Hence $d_{K_{t}} \gg t^{2}$, and Assumption 4.1 is verified. We have proved the following Proposition.

Proposition 8.2 If $27 t^{4}+1$ is cubic free, $R_{t} \ll \log d_{K_{t}}$. 
As described in Section 4, we consider a set $L(X)$ of cube-free integers

$$
L(X)=\left\{\frac{X}{2}<t<X: 27 t^{4}+1 \text { cube-free and } t \equiv t_{M}(\bmod M)\right\}
$$

We prove the following lemma, which is a direct consequence of [8, p. 69].

Lemma 8.3 Let $f(x)$ be an irreducible polynomial of degree $d \geq 3$ in $\mathbb{Z}[x]$. Let $M$ be a positive integer and $\operatorname{gcd}(a, M)=1$. Suppose that if $p \mid M$, then $f(a) \not \equiv 0(\bmod p)$. Let $N(X, f, M)$ be the number of integers $1 \leq n<X$ and $n \equiv a(\bmod M)$, with the property that $f(n)$ is $(d-1)$-free. Then

$$
N(X, f, M)=C(M) \frac{X}{M}+O\left(\frac{X}{M}\left(\log \frac{X}{M}\right)^{\frac{2}{d+1}-1}\right),
$$

where $C(M)=\prod_{p \nmid M}\left(1-\rho\left(p^{d-1}\right) / p^{d-1}\right)$, and $\rho\left(p^{k}\right)$ is the number of solutions for $f(x) \equiv 0\left(\bmod p^{k}\right)$.

Proof Let $n=M m+a$, and $g(m)=f(M m+a)$. Then $1 \leq m<X / M$, and $g(x)$ is an irreducible polynomial of degree $d$. Hooley [8, p. 69] showed that the number of $1 \leq m<X / M$ with the property that $g(m)$ is $(d-1)$-free is

$$
C(M) \frac{X}{M}+O\left(\frac{X}{M}\left(\log \frac{X}{M}\right)^{\frac{2}{d+1}-1}\right),
$$

where

$$
C(M)=\prod_{p \nmid M}\left(1-\frac{\rho^{\prime}\left(p^{d-1}\right)}{p^{d-1}}\right),
$$

and $\rho^{\prime}\left(p^{d-1}\right)$ is the number of solutions for $g(m) \equiv 0\left(\bmod p^{d-1}\right)$. If $p \mid M, g(m) \equiv$ $f(a) \not \equiv 0(\bmod p)$. Hence $\rho^{\prime}\left(p^{d-1}\right)=0$. If $p \nmid M$, then since $M m+a \equiv 0$ $\left(\bmod p^{d-1}\right)$ has a unique solution $\bmod p^{d-1}, \rho^{\prime}\left(p^{d-1}\right)=\rho\left(p^{d-1}\right)$. Our result follows.

Note that by the definition of $M, t_{M}$, if $p \mid M$, then $c_{f} \leq p \leq y, t_{M} \equiv t_{p}(\bmod p)$, and $p$ splits completely in $\widehat{K}_{t_{p}}$. Then $27\left(M m+t_{M}\right)^{4}+1 \equiv 27 t_{p}^{4}+1 \not \equiv 0(\bmod p)$. Hence, $\rho(p)=0$. This implies that $\rho\left(p^{3}\right)=0$. If $p \nmid M$, by Nagell [15, p. 87], $\rho\left(p^{3}\right)=\rho(p) \leq 4$. So

$$
\prod_{p \nmid M}\left(1-\frac{\rho\left(p^{3}\right)}{p^{3}}\right) \gg \prod_{p \nmid M}\left(1-4 p^{-3}\right) \geq \prod_{p \nmid M}\left(1-p^{-3}\right)^{5} \geq \zeta(3)^{-5} .
$$

Hence, by the above lemma,

$$
|L(X)|=C(M) \frac{X}{2 M}+O\left(\frac{X}{M\left(\log \frac{X}{M}\right)^{\frac{3}{5}}}\right) \text { and }|L(X)| \gg X^{1-\epsilon} .
$$

In the remark below, we use the recent result of Heath-Brown [9] to obtain a better error term in $|L(X)|$. 
Here different $t_{1}, t_{2} \in L(X)$ may give rise to the same $L$-function. We need to know the locations of the roots more precisely to distinguish the L-functions. By applying Lemma 5.3 with $\alpha=4 t+1.4 t i$, for sufficiently large $t$, we find a complex root inside the circle of radius $0.03 \sqrt{2} t$ centered at $4.015 t+1.385 t i$. Again by applying Lemma 5.3 with $\alpha=0.23 i / t$, for sufficiently large $t$, we find a complex root inside the circle of radius $0.0115 / t$ centered at $0.2415 i / t$.

We order the roots of $f(x, t)$ in the following way. Let $\theta_{t}^{1}$ be the root near the origin whose imaginary part is positive and $\theta_{t}^{2}=\overline{\theta_{t}^{1}}$. Let $\theta_{t}^{3}$ be the other root whose imaginary part is positive and $\theta_{t}^{4}=\overline{\theta_{t}^{3}}$. Let $\tau$ be the complex embedding of $K_{t}$ that maps $\theta_{t}^{1}$ to $\theta_{t}^{3}$.

If $t_{1}, t_{2} \in L(X)$ give rise to the same $L$-function, $\mathbb{Q}\left(\theta_{t_{1}}^{1}\right)$ and $\mathbb{Q}\left(\theta_{t_{2}}^{1}\right)$ are isomorphic, since they are quartic fields. Hence $\mathbb{Q}\left(\theta_{t_{1}}^{1}\right)=\mathbb{Q}\left(\theta_{t_{2}}^{j}\right)$ for some $1 \leq j \leq 4$. Assume that 33 different $t_{1}, t_{2}, \ldots, t_{33}$ give rise to the same $L$-function. Then we can see that there are at least nine $t_{i_{1}}, t_{i_{2}}, \ldots, t_{i_{9}}$ with $\mathbb{Q}\left(\theta_{t_{i_{1}}}^{k}\right)=\mathbb{Q}\left(\theta_{t_{i_{2}}}^{k}\right)=\cdots=\mathbb{Q}\left(\theta_{t_{i_{9}}}^{k}\right)$ for some $1 \leq k \leq 4$. Without loss of generality, we assume that $k=1$. Then there are at least two $t_{i_{l}}, t_{i_{m}}$ such that $\tau: \theta_{t_{i_{l}}}^{1} \rightarrow \theta_{t_{i}}^{3} ; \tau: \theta_{t_{i_{m}}}^{1} \rightarrow \theta_{t_{i_{m}}}^{3}$. Now we further assume that $0.55 X<t<X$. Then

$$
N\left(\theta_{t_{i_{l}}}^{1}-\theta_{t_{i_{m}}}^{1}\right)<\left(\frac{0.253}{0.55 X}\right)^{2} \times(4.277 X-4.199 \times 0.55 X)^{2} \approx 0.8232840<1 .
$$

Since $\theta_{t_{i_{l}}}^{1}-\theta_{t_{i_{m}}}^{1} \neq 0$, it induces a contraction. So there are at most $32 t$ 's giving rise to the same $L$-function. Hence Assumption 4.2 holds. Let $\widetilde{L}(X)$ be the set of distinct $L$-functions coming from $L(X)$. By the above argument, we have

$$
X^{1-\epsilon} \ll|\widetilde{L}(X)| \ll X
$$

Let $L(s, \rho, t)=\zeta_{K_{t}}(s) / \zeta(s)$. Then $\rho$ is equivalent to a twist of $\operatorname{Sym}^{2}(\sigma)$ by a character, where $\sigma$ is the 2-dimensional representation of $\widetilde{A}_{4} \simeq S L_{2}\left(\mathbb{F}_{3}\right)$. Hence $\rho$ is modular and $L(s, \rho, t)$ is the cuspidal automorphic $L$-function of $G L_{3} / \mathbb{Q}$. See [2] for the details. We have the following theorem.

Theorem 8.4 There is a constant $c>0$ such that there exist $K \in \mathfrak{K}\left(4, A_{4}, 0,2\right)$ with arbitrary large discriminant $d_{K}$ for which

$$
h_{K}>c d_{K}^{\frac{1}{2}} \frac{\left(\log \log d_{K}\right)^{3}}{\log d_{K}} .
$$

Remark 8.5 Let $M \ll X^{\delta^{\prime}}$ with $0<\delta^{\prime}<\delta$, where $\delta$ is the constant in [9]. Then we can show that

$$
|L(X)|=\prod_{p \nmid M}\left(1-\frac{\rho\left(p^{3}\right)}{p^{3}}\right) \frac{X}{M}+O\left(X^{1-\delta}\right),
$$

where $\rho\left(p^{3}\right)$ is the number of solutions to $27 t^{4}+1 \equiv 0\left(\bmod p^{3}\right)$. 
Remark 8.6 Let $f(x, t)$ be a family of quintic polynomials and let $\theta_{t}$ be a root, and $K_{t}=\mathbb{Q}\left[\theta_{t}\right]$. Let $\widehat{K}_{t}$ be the Galois closure of $K_{t}$. Assume that $G=\operatorname{Gal}\left(\widehat{K}_{t} / \mathbb{Q}\right)$ is isomorphic to $A_{5}$ and that $K_{t}$ has signature $(1,2)$. We also assume that $f(x, t)$ gives rise to a regular Galois extension over $\mathbb{Q}(t)$. For example,

$f(x, t)=x^{5}-5\left(5 t^{2}-1\right) x^{4}-\left(4\left(5 t^{2}-1\right)\right)^{4}$ or $f(x, t)=x^{5}+5\left(5 t^{2}-1\right) x+4\left(5 t^{2}-1\right)$

satisfies those properties. Then $G$ has a subgroup $H$ isomorphic to $A_{4}$ such that $\widehat{K}_{t}^{H}=K_{t}$. Let Ind ${ }_{H}^{G} 1_{H}=1_{G} \oplus \rho$, where $\rho$ is the 4-dimensional representation of $A_{5}$. Then

$$
L(s, \rho, t)=\frac{\zeta_{K_{t}}(s)}{\zeta(s)}
$$

Now by [11, p. 498], $\rho$ is equivalent to a twist of $\sigma \otimes \sigma^{\tau}$ by a character, where $\sigma, \sigma^{\tau}$ are the icosahedral 2-dimensional representations of $\widetilde{A}_{5} \simeq S L_{2}\left(\mathbb{F}_{5}\right)$. Since $K_{t}$ is not totally real, $\sigma$ and $\sigma^{\tau}$ are odd. Hence by [10, Corollary 10.2], $\sigma, \sigma^{\tau}$ are modular, i.e., they are attached to cuspidal representations $\pi, \pi^{\tau}$ of $G L_{2} / \mathbb{Q}$. By [18], the functorial product $\pi \otimes \pi^{\tau}$ is a cuspidal representation of $G L_{4} / \mathbb{Q}$. Hence $L(s, \rho, t)$ is a cuspidal automorphic $L$-function of $G L_{4} / \mathbb{Q}$. In particular, we can prove that there exists an arbitrarily large $t$ such that $L(1, \rho, t) \gg\left(\log \log d_{K_{t}}\right)^{4}$. Unfortunately, for the above polynomials, numerical calculation shows that the regulator of $K_{t}$ seems large. We have not been able to find a family of $A_{5}$ quintic polynomials with small regulators, of size $(\log t)^{2}$.

\section{References}

[1] N. C. Ankeny, R. Brauer, and S. Chowla, A note on the class-numbers of algebraic number fields. Amer. J. Math. 78(1956), 51-61. http://dx.doi.org/10.2307/2372483

[2] P. J. Cho, The strong Artin conjecture and number fields with large class numbers. Q. J. Math., to appear.

[3] P. J. Cho and H. H. Kim, Dihedral and cyclic extensions with large class numbers. J. Th. Nombres Bordeaux, to appear.

[4] F. Calegari, The Artin Conjecture for some $S_{5}$-extensions. arxiv:1112.1152v1

[5] H. Cohen, A course in computational algebraic number theory. Graduate Texts in Mathematics, 138, Springer-Verlag, Berlin, 1993.

[6] W. Duke, Extreme values of Artin L-functions and class numbers. Compositio Math. 136(2003), no. 1, 103-115. http://dx.doi.org/10.1023/A:1022695505997

[7] R. C. Daileda, Non-abelian number fields with very large class numbers. Acta Arith. 125(2006), no. 3, 215-255. http://dx.doi.org/10.4064/aa125-3-2

[8] C. Hooley, Applications of sieve methods to the theory of numbers. Cambridge Tracts in Mathematics, 70, Cambridge University Press, Cambridge, 1976.

[9] D. R. Heath-Brown, Powerfree values of polynomials. arxiv:1103.2028.

[10] C. Khare and J. P. Wintenberger, Serre's modularity conjecture. I. Invent. Math. 178(2009), no. 3, 485-504. http://dx.doi.org/10.1007/s00222-009-0205-7

[11] H. H. Kim, An example of non-normal quintic automorphic induction and modularity of symmetric powers of cusp forms of icosahedral type. Invent. Math. 156(2004), no. 3, 495-502. http://dx.doi.org/10.1007/s00222-003-0340-5

[12] N. Klingen, Arithmetical similarities. Oxford Mathematical Monographs, Oxford Science Publications, The Clarendon Press, Oxford University Press, New York, 1998.

[13] E. Kowalski and P. Michel, Zeros of families of automorphic L-functions close to 1. Pacific J. Math. 207(2002), no. 2, 411-431. http://dx.doi.org/10.2140/pjm.2002.207.411

[14] H. L. Montgomery and P. J. Weinberger, Real quadratic fields with large class numbers. Math. Ann. 225(1977), no. 2, 173-176. http://dx.doi.org/10.1007/BF01351721 
[15] T. Nagell, Introduction to number theory. Second ed., Chelsea Publishing Co., New York, 1964.

[16] W. Narkiewicz, Elementary and analytic theory of algebraic numbers. Second ed., Springer-Verlag, Berlin, PWN-Polish Scientific Publisher, Warsaw, 1990.

[17] N. Obreschkoff, Verteilung und Berechnung der Nullstellen reeller Polynome. Deutscher Verlag der Wissenschaften, Berlin, 1963

[18] D. Ramakrishnan, Modularity of the Rankin-Selberg L-series, and multiplicity one for SL(2). Ann. of Math. 152(2000), no. 1, 45-111. http://dx.doi.org/10.2307/2661379

[19] S. Sasaki, On Artin representations and nearly ordinary Hecke algebras over totally real fields. I. http://www.cantabgold.net/users/s.sasaki.03/

[20] J. Sándor, D. Mitrinović, and B. Crstici, Handbook of number theory. I. Springer, Dordrecht, 2006.

[21] A. M. Schöpp, Fundamental units in a parametric family of not totally real quintic number fields. J. Théor. Nombres Bordeaux 18(2006), no. 3, 693-706. http://dx.doi.org/10.5802/jtnb.567

[22] J.-P. Serre, Topics in Galois theory, Research Notes in Mathematics, 1, A K Peters, Ltd., Wellesley, MA, 2008.

[23] J. H. Silverman, An inequality relating the regulator and the discriminant of a number field. J. Number Theory 19(1984), no. 3, 437-442. http://dx.doi.org/10.1016/0022-314X(84)90082-9

[24] Y. Zhang, L-functions in number theory. Ph.D. Thesis, University of Toronto, 2009, ProQuest LLC, Ann Arbor, MI, 2010

Department of Mathematics, University of Toronto, Toronto, ON M5S 2E4

e-mail: jcho@math.toronto.edu

Department of Mathematics, University of Toronto, Toronto, ON M5S 2E4

and

Korea Institute for Advanced Study, Seoul, Korea

e-mail: henrykim@math.toronto.edu 